\title{
Three-dimensional optical data storage through multi-photon confocal microscopy and imaging
}

\author{
William Dallari ${ }^{a}$, Marco Scotto $^{a}$, Marco Allione ${ }^{a, *}$, Elena Samoylova ${ }^{a}$, Francesca Pignatelli ${ }^{a}$, \\ Roberto Cingolani $^{\mathrm{a}}$, Athanassia Athanassiou ${ }^{\mathrm{b}}$, Alberto Diaspro ${ }^{\mathrm{a}}$ \\ a Italian Institute of Technology, Genova I-16163, Italy \\ ${ }^{\mathrm{b}}$ Center for Biomolecular Nanotechnologies - Italian Institute of Technology, Arnesano (LE) I-73010, Italy
}

\section{A R T I C L E I N F O}

\section{Article history:}

Received 17 September 2010

Received in revised form 3 May 2011

Accepted 3 May 2011

Available online 14 May 2011

\section{Keywords:}

Optical data storage

Multiphoton confocal microscopy

\begin{abstract}
A B S T R A C T
Three dimensional optical data storage is one of the most promising tools to respond to the always growing demand for high data storage capacity. Here, we focused a femtosecond laser source by means of a confocal microscope onto different transparent recording media. The purpose of the study is to probe the capability of the system to independently address different data layers within the storage medium achieving thus three dimensional data storage. We demonstrated the possibility to write superposed independent layers of data due to either multiphoton excitation or to local optical breakdown and the performances observed in the different types of media used are compared.
\end{abstract}

(c) 2011 Elsevier B.V. All rights reserved.

\section{Introduction}

The long time ongoing quest for higher performance electronic devices and higher computational power systems has triggered the strong demand for the storage of the growing amounts of data. The ideal storage medium should have the capability of a high data density and transfer speed, a high durability in time and different environmental conditions, and ease of implementing a production line. Particularly appealing, not yet fundamental for all the foreseen application, is the capability to erase and rewrite the data many times in the device. Among the possible applications of a data storage device, optical data storage is certainly a very promising and already well established one.

Traditional optical data storage is hampered by the amount of the recorded data due to two limiting factors: a physical one given by diffraction limit, close to its highest performance (Blu-ray ${ }^{\circledR}$ ), and a geometrical one given by the two-dimensional properties of writing and readout techniques in the typical technological implementation.

Within this framework, the natural step forward is given by the investigation on the feasibility of a three-dimensional multi-layered optical memory prototype [1]. The major challenges in such a device are given by the non-destructive readout and the interlayer cross talk in the third dimension.

\footnotetext{
* Corresponding author.

E-mail address: marco.allione@iit.it (M. Allione).
}

Several different configurations and setups have been proposed [2] and, among them, infrared multi-photon interaction has been identified as one of the most promising writing technique, due to its intrinsic 3D spatial localization ensuring high density and negligible cross talk at the very same time [3]. On the other hand, the new nanocomposite materials represent the key for a durable and low cost optical memory.

In this work, we present the proof of principle for a three dimensional optical data storage system based on different type of materials by using a conventional multiphoton microscopy architecture [4] coupled with a femtosecond laser source. We hereby present results obtained in reversible materials, i.e. in materials in which the photo-induced transition can be reversed upon irradiation with light at particular wavelength, and in irreversible ones. While the former materials are promising for read-write-erase memories, the latter, thanks to their high stability and durability in time, could prove to be more suitable for Write-Once-ReadMany (WORM) data storage systems.

Upon excitation of the sample above a given intensity threshold, the focused laser beam induces a modification of the local optical properties of the sample in a small region determined by the beam waist. Such a modification is due to either the insurgence of multiphoton processes or to the achievement of an activation threshold for photochemical or photothermal processes, depending on the material used and on the beam intensity. For all the materials used, the values of excitation intensity and exposure times are reported, the linear or nonlinear behavior of the photoconversion is discussed and a comparison of the results obtained from the different materials is presented. 


\section{Materials and methods}

Several materials (nanorods, photochromic molecules, semiconductors) can be embedded in a host material to allow three-dimensional writing of information by two-photon absorption within the storage medium. Our attention was focused on diarylethenes photochromic molecules incorporated in different polymers [5] and a commercial semiconductor doped glass [6]. We also irradiated an undoped polymer with a pulsed laser towards $3 \mathrm{D}$ recording $[7,8]$.

\subsection{Reversible photocyclization of aromatic compounds}

Diarylethenes belong to the thermally irreversible group of photochromic compounds. This property, added to their high resistance to fatigue which allows performing several write-read-erase cycles, makes diarylethenes-based systems very attractive for optical data storage [9]. The specific molecule that we utilized for recording information by two-photon absorption was 1,2-Bis[2methylbenzo[ $b]$ thiophen-3-yl]-3,3,4,4,5,5-hexafluoro-1-cyclopentene (MBT) embedded in different polymer matrices (see Section S1 of Supporting Information for details).

\subsection{Commercial semiconductor-doped glass}

The sample is a $3 \mathrm{~mm}$ thick long pass filter (OG-570, Schott AG) placed directly on the microscope focal plane.

\subsection{Undoped polymer}

A chloroform solution of PMMA $(150 \mathrm{mg} / \mathrm{ml})$ was cast over a microscope cover glass and dried in an oven at $40{ }^{\circ} \mathrm{C}$ for $1 \mathrm{~h}$ to remove solvent residual.

For a general description of the optical setup used see paragraph S2 in the Supporting Information.

\section{Results and discussion}

Polymer-photochromic molecule blends seem to be a promising tool for erasable and rewritable memories, since the material can be photoconverted several times [5]. In particular, diarylethenes photochromic molecules seem to be particularly interesting thanks to their thermal stability and excellent fatigue resistance.

All polymers doped with MBT showed a photo-conversion when illuminated by $720 \mathrm{~nm}$ pulsed laser light. The result of the irradiation is given by the formation of an area where most all the molecules are in a photo-induced state having a strong fluorescence emission in the orange-red range when excited at $405 \mathrm{~nm}$ [10]. As neither the polymer nor the photochromic molecule has an absorption band at this wavelength $[11,12]$, the process must be originated by a two-photon interaction as confirmed by the behavior of the fluorescence emission versus the laser power shown in the inset of Fig. 1. MBT preserves its photochromism and the back-conversion is highly efficient when irradiated at $515 \mathrm{~nm}$.

In order to probe in such material the capability to address different data layers series of spots separated by $5 \mu \mathrm{m}$ in the $\mathrm{z}$ direction (depth) have been written in the volume of the recording medium by the two-photon writing process (Fig. 1). This $5 \mu \mathrm{m}$ spacing did not produce interlayer cross talk. The required intensities and exposure times to perform this experiment in this material are reported in Table 1.

A problem to overcome before commercialization of photochromic-based compounds is destructive readout. In fact, for almost all the photochromic molecules the absorption band of the read and erase states are so close to lead to the erase of the information

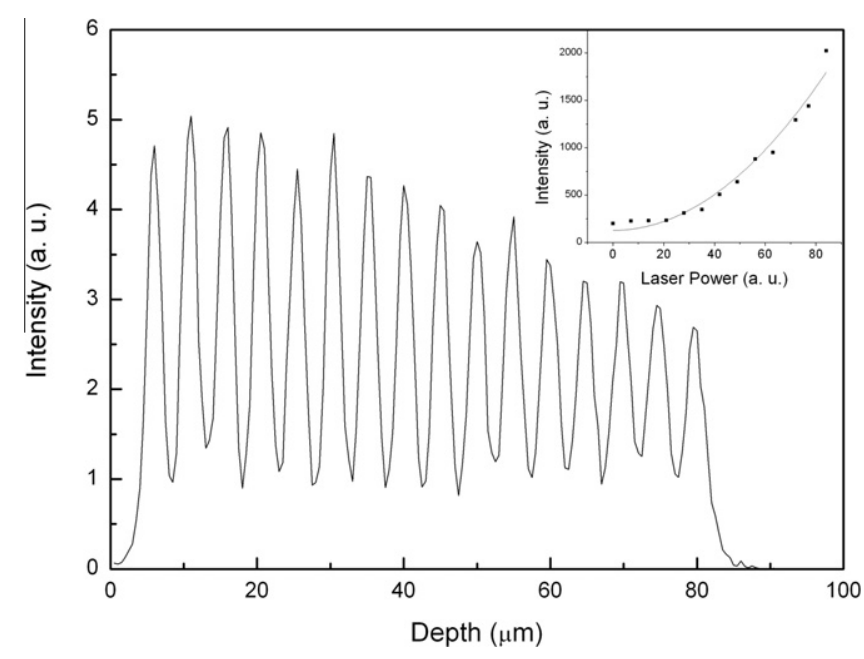

Fig. 1. Intensity plot of the luminescence signal for a sequence of isolated spots aligned along the $z$ direction. The sample is a blend of MBT in PEMMA. The vertical displacement of the dots is $5 \mu \mathrm{m}$ while the conditions used to record the spots and to collect the luminescence are the same as in Fig. S1 in the Supporting Information. The inset shows the photoluminescence intensity of single spots as a function of the laser intensity used for recording. The quadratic fit to the data $(R=0.972)$ clearly demonstrate the nonlinear dependence of the signal and thus the multiphoton spatially localized nature of the writing process $\left(\lambda_{\text {exc }}=405 \mathrm{~nm}\right.$, detection range $=575-640 \mathrm{~nm})$.

Table 1

Comparison between the parameters for single spot photoconversion in the different samples employed in our work. Last line density is calculated from vertical and horizontal dimensions of the spots.

\begin{tabular}{llll}
\hline & Longpass filter & Undoped polymer & Doped polymer \\
\hline Type & WORM & WORM & RW \\
$\lambda_{\mathrm{W}}(\mathrm{nm})$ & 720 & 720 & 720 \\
$\lambda_{\mathrm{R}}(\mathrm{nm})$ & 515 & 405 & 405 \\
$t_{\mathrm{w}}(\mathrm{ms})$ & 50 & 50 & 50 \\
$P_{\mathrm{W}}(\mathrm{mW})$ & 13.4 & 13.4 & 13.4 \\
$\sigma_{\mathrm{xy}}, \sigma_{\mathrm{z}}(\mu \mathrm{m})$ & $2.2,8.5$ & $1,3.1$ & $0.9,2.5$ \\
Density $\left(\mathrm{Gbit} / \mathrm{cm}^{3}\right)$ & 24 & 322 & 500 \\
\hline
\end{tabular}

stored after repetitive reading. For this reason, readout systems not based on luminescence such as detecting small changes in refractive index in the infrared, have already been proposed [13], as well as methods based on the excitation of the luminescence via resonant energy transfer from other chromophores [14]. Alternatively also the presence of different host polymers can affect the stability of the memory under readout conditions, and some studies are ongoing in our group to test this particular aspect [15].

On the other side, in contrast to the rewritable storage materials, WORM media could play an important role as inexpensive massive data warehouse systems. We have hereby demonstrated the proof of principle on some different materials.

The commercial longpass Schott glass filter that we used contains a dispersion of absorbers that fluoresce in the $575-640 \mathrm{~nm}$ range when excited by $405 \mathrm{~nm}$ laser. We tried to inhibit this light emission by photobleaching diffraction-limited spots using the same $720 \mathrm{~nm}$ pulsed laser source used for irradiating photochromic compounds. The decrease of the fluorescence intensity from its original value as function of the NIR laser power was measured and an example is shown in Fig. 2. Results did not indicate the presence of any nonlinear behavior in the writing process, with the presence of a saturation effect for the highest intensities used. Without the peculiar high localization provided by the multiphoton process, the vertical compactness of data is inevitably reduced. In fact, it was necessary to vertically displace at least $15 \mu \mathrm{m}$ the 


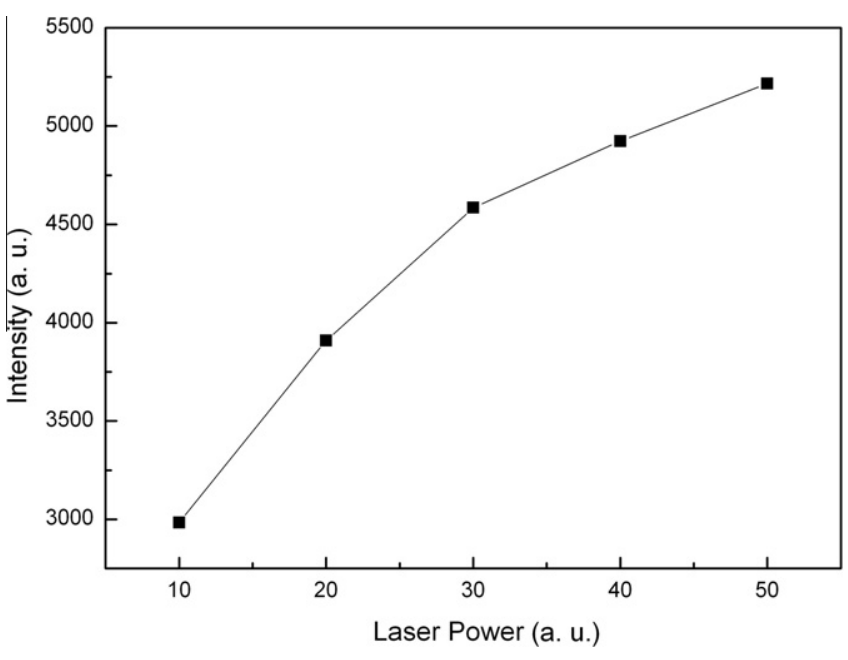

Fig. 2. Depletion of the luminescence intensity as a function of the laser power used for irradiation $\left(\lambda_{\mathrm{exc}}=405 \mathrm{~nm}\right.$, detection range $=575-640 \mathrm{~nm}$ ). The sample is a commercial semiconductor-doped glass (OG-570, Schott AG).

data layers in this samples in order to avoid data cross-talk. The full details of the writing and reading processes are summarized in Table 1.

Alternatively, some groups have recently demonstrated that an undoped polymer can be a suitable medium for 3D storage. Some papers $[16,17]$ have reported that microvoids can be generated in polymer material using ultrashort pulsed laser. The strong electromagnetic field induces ionization in the focal volume that creates damage and a strong photoluminescence emission in the visible range. This photoluminescence change therefore can be used for read-only optical data storage. We were able to record single spots inside a pure PEMMA film by the use of femtosecond pulsed irradiation at $720 \mathrm{~nm}$ achieving a 3D memory, whose recorded bits were detected by means of their photoluminescence (for an example of spots written at different power levels see Fig. S2 of the Supporting Information). Also for this case, the data on the obtained spots are listed in Table 1.

From the analysis of the different results in Table 1 it is clear that the results obtained are much better in the polymer and in the polymeric blend with photochromic molecules because of the nonlinear nature of the excitation in this case, but most likely also to a lower spherical aberration with respect to the doped glass, in which the refractive index mismatch with respect to the water (for which our microscope is optimized) is higher ( $n=1.51$ for this glass in the visible range [18]). Quite surprisingly, the intensity required for optimal performance in the two polymeric materials is the same. The exposure time used here is pretty long compared to the one required for high data rate application, but here it was kept the same on all samples for the sake of comparison. On the other side, while the strongest change in photoluminescence from the unperturbed samples is peaked in the red-orange part of the visible spectrum for the photochromic material, it is mainly concentrated in the green-blue region for the undoped polymer. In the doped polymer then, it might be expected the presence of both photoconversions and this is actually what we observed. This does not affect the device working principle, since the two luminescence contributions can be spectrally well distinguished. In this case also, it seems that the permanent transformation of the polymer is less evident than for the case of the undoped material, since the presence of the photochromic molecules absorb part of the incoming radiation increasing a bit the threshold for the permanent damage. It is in fact quite evident from Fig. S2, where spots in the pure polymer at variable intensities are reported, that this process is strongly intensity-dependent and the size of the obtained spot, despite it could be quite small, varies abruptly with intensity around the threshold. This represent an issue also for the uniformity of the material used, in which even small fluctuation of the optical properties strongly affect the size of the spots obtained. A perfect control of the material homogeneity is then crucial in this case.

It seems then that the three dimensional writing in photochromic compound can meet the requirements for a high density optical data storage. Nevertheless the problem of destructive readout remains. Such problem could be partially overcome by choosing the appropriate host material. This option, which is currently under investigation in our group, is not much investigated in literature, although it is well known that the physical properties of the host matrix can affect the photoconversion processes $[5,19]$.

With regards to the WORM materials used, it looks like that a better choice of the materials has to be done to meet the requirement for efficient optical data storage. The undoped polymers used are too sensitive to material uniformity and experimental conditions. The semiconductor doped glasses, despite perhaps the resolution could be increased with the design of the proper focusing optics, show a behavior that excludes nonlinear processes and that better vertically confines the bit. Moreover it has to be added that, since the light used for readout is absorbed by the medium, this material showed a quite strong destructive read out problem. A material doped with nanostructured light absorbers, acting as localized catalysers of the phototransition, might be in our opinion a good way to minimize the volume of a single voxel.

\section{Conclusions}

In conclusion, we have successfully coupled conventional confocal microscopy with femtosecond laser source to realize threedimensional optical erasable-rewritable and WORM memory for data storage applications. Although further research and development of new detection techniques are needed to overcome destructive readout, from our preliminary tests it turned out that diarylethene-based materials seem to be a promising candidate for erasable and rewritable devices.

Concerning WORM devices, luminescence quenching via laser excitation seems to be a promising and easy-to-implement technique despite the materials we tested here seem not to be the best choice in this direction. It is possible that the wide variety of nanostructures that have been chemically synthesized in the last years may play a role in this kind of application, as recent results in literature seem to indicate [20].

\section{Appendix A. Supplementary data}

Supplementary data associated with this article can be found, in the online version, at doi:10.1016/j.mee.2011.05.006.

\section{References}

[1] D.A. Parthenopoulos, P.M. Rentzepis, Science 245 (1989) 843-845.

[2] A. Toriumi, S. Kawata, M. Gu, Opt. Lett. 23 (1998) 1924-1926. and references therein.

[3] A.S. Dvornikov, E.P. Walker, P.M. Rentzepis, J. Phys. Chem. A 113 (2009) 1363313644.

[4] A. Diaspro et al., Quart. Rev. Biophys. 38 (02) (2005) 97-166.

[5] E. Samoylova et al., in: Conference Proceedings of IEEE NANO 2010, Seoul, Korea, 2010

[6] G. Banfi et al., Phys. Rev. B 50 (8) (1994) 5699-5702.

[7] Z. Nie, H. Lee, H. Yoo, et al., Appl. Phys. Lett. 94 (2009) 11912-1-11912-3.

[8] K. Yamasaki et al., Appl. Phys. Lett. 76 (8) (2000) 1000-1002.

[9] M. Irie, Chem. Rev. 100 (5) (2000) 1685-1716.

[10] M. Kim et al., Opt. Mater. 21 (2002) 271-274.

[11] J. Hongfang, Z. Lixin, J. Rare Earth 27 (5) (2009) 786-789.

[12] M. Irie et al., J. Am. Chem. Soc. 116 (22) (1994) 9894-9900.

[13] C. Corredor et al., Chem. Mater. 19 (2007) 5165-5173. 
[14] T. Tsujioka et al., Appl. Phys. Lett. 78 (16) (2001) 2282-2284.

[15] E. Samoylova et al., Adv. Funct. Mater. (2010).

[18] Technical Datasheet, Schott AG, Germany.

[16] D. Day, M. Gu, Appl. Phys. Lett. 80 (13) (2002) 2404-2406.

[19] J.-S. Lin, Eur. Polym. J. 39 (2003) 1693.

[17] Katayama et al., J. Polym. Sci. B 40 (2002) 537-544.

[20] P. Zijlstra et al., Nature 459 (2009) 410-413. 